\section{Molecular Recognition}

MS11.01.01 COMPLEXES OF FULLERENES WITH HOST MOLECULES. Jerry L. Atwood, Department of Chemistry, University of Missouri, $6091 \mathrm{~S}$. College Ave., Columbia, MO 65211

Because of the shape and symmetry of $\mathrm{C}_{60}$. crystallographic structure determinations often involve problems, such as disorder. Host-guest complexes of $\mathrm{C}_{60}$ and calixarenes and cyclotriveratrylenes (CTV) have provided ways of ordering the fullerene. In this discussion I will address the strategy for the complexation of $\mathrm{C}_{60}, \mathrm{C}_{70}$, and higher fullerenes. Specifically, attention will be paid to the matching of symmetry of the fullerene with the host molecule.

MS11.01.02 REACTIVITYAND STRUCTURE OF CHMRAL CRYSTAL. Fumio Toda, Department of Applied Chemistry, Faculty of Engineering, Ehime University, Matsuyama, Ehime 790. Japan

We have been studied the relationship between reactivity and structure of inclusion chiral crystal prepared from chiral host and achiral guest. In this microsymposium, we present some interesting difference of selectivity of photoreaction in the chiral crystal prepared by recrystallization of chiral host and achiral guest compounds from solvent and that prepared by mixing of powdered both components in the solid state. In most cases, photoreaction of the host-guest inclusion crystal prepared by recrystallization of chiral host and achiral guest from solvent and that prepared by mixing powdered both components gave the optically active product of the same chirality. X-ray analysis of the inclusion crystal prepared by the recrystallization showed that achiral guest molecules are arranged in a chiral form in the inclusion crystal. In a special case, however, photoirradiation of the host-guest inclusion crystal prepared by recrystallization of an achiral guest and chiral host compound from solvent gave $(+)$-chiral photo-reaction product. On the other hand, the same reaction of the inclusion crystal prepared by mixing the both in the solid state gave (-)-chiral photoreaction product.

The very interesting difference of inclusion behavior by chiral host and racemic host was found. Chiral and racemic 7-bromo1,4,8-triphenyl-2,3-benzo[3.3.0]octa-2,4,7-trien-6-one include 4picoline and 2-picoline, respectively.

MS11.01.03 CRAFTING POROUS CRYSTALLINE NETWORKS IN MOLECULAR BASED SOLIDS. Israel Goldberg, School of Chemistry, TelAviv University, 69978 Ramat-Aviv, Israel

The versatility of porphyrin-based metallomacrocycles as crystalline hosts appears to be unequaled due to the large size, high symmetry, rigidity and thermal stability of the molecular framework. It is feasible to tailor their shape and functionality features by organic synthesis, and thus affect the microstructure of the resulting assembly. In this study we demonstrate that a suitable functionalization of the metallomacrocyclic framework with polarized aryl groups can be used to develop simple chemical models of self-assembly via weak intermolecular forces, and to control the spontaneous built-up of the porphyrin lattice by molecular recognition properties of the respective sensor groups. The strength and multiplicity of the noncovalent interactions are expected to act in concert to reinforce directional preferences and dominate the crystal field. In the resulting crystalline materials the molecular building blocks are incorporated into pseudo-rigid polymeric arrangements with a porous architecture.

Our preparative efforts focused on the design of several new classes of hollow crystalline networks in which the metallomacrocyclic units are linked into multidimensional poly- meric architectures via metal-ligand coordination, hydrogenbonding, dipolar association, halogen-halogen interaction, $\pi-\pi$ stacking and $\mathrm{C}-\mathrm{H} \cdot \pi \cdot \mathrm{\pi} \cdot \mathrm{contacts}$. The size and shape characteristics of the pore structure in these networks are determined by the nature of the particular supramolecular synthon involved in steering the intermolecular self-assembly. Most of the porphyrin-based solids have a strong tendency to incorporate molecular guest components of complementary shape into the lattice, and thus provide novel types of potential solid state receptors for isolation, separation, transport, exchange and controlled release of molecular entities. The structural and inclusion features of the various motifs will be discussed in some detail.

MS11.01.04 MODELLING MICELLAR AGGREGATION OF BILE SALTS. Sofia Candeloro De Sanctis, Facolta' di Medicina, Dipartimento di Chimica, Universita' di Roma 'La Sapienza"

The bile salts anions are amphiphilic steroid-like molecules with a rigid backbone of four fused rings from which three methyl and a variable number of hydroxyl groups are protruding. The molecules have flexible side chains of variable length and chemi$\mathrm{cal}$ constitution. In the physiological medium they interact through micellar aggregation, with important molecules like cholesterol, bilirubin, phospholipids, fatty acids. In most of the crystals we have studied so far they aggregate into very stable helical structures.

The helices are stabilized by an extended network of hydrogen bonds, as well as ion-ion and ion-dipole interactions. Molecules with different side chains and counterions can give rise to very similar packing motifs with similar hydrogen bonding schemes, indicating the important role of the hydrogen bonds for such structures.

The outer surfaces of the helices in the crystals have large non polar regions. However, for some of the bile salts we have evidences indicating the stability of the helical structures in the aqueous solutions, suggesting their presence possibly in the physiological medium as well. We are, therefore, using the helical arrangements found in the crystals as models for the micellar aggregation of bile salts and for their possible selective interactions with the molecules of the biological medium.

MS11.01.05 MOLECULAR RECOGNITION OF DIHOMOOXACALIX[4]ARENE IN THE CRYSTAL STRUCTURES OF $(1: 1)$ COMPLEXES WITH XYLENE ISOMERS. Ken-ichi Tomita",Kenji Suzuki\#,Isao Nakanishi+, Anthony E. Armah*:Zouhair Asfari@, Jacques Vicens@, *Faculty of Pharmaceutical Sciences, Osaka University, Suita, Osaka 565, \#Dainihon Pharmaceutical Co., Enokicho, Suita, Osaka 564, +Fujisawa Pharmaceutical Co., Kashima, Yodogawa-ku, Osaka 532, Japan, @Laboratoire de Chimie Minerale et Analytique, E.H.I.C.S., Strasbourg, France

Dihomooxacalix [4]arenes (1) are macrocycles made from four phenolic units meta-linked by three methylene and one methylene oxy-methylene bridges at positions ortho to the hydroxy groups, and the hydrogen atom at the position para to the hydroxy group of phenolic residues was replaced with bulky aliphatic groups ( $\mathrm{p}-$ isopropyl or p-tert-butyl). Recently, we have reported our results on the isomer separation of the xylenes by extractive crystallization with calix[4]arenes including dihomooxacalix[4]arene(2), and p-isopropyldihomooxacalix[4]arene extracted oxylene with $84 \%$ selectivity while para-xylene with only $16 \%$ selectivity. This fact may indicate the difference and strength of molecular recognition of xylene isomers by dihomooxacalix[4]arene. Therefore, we solved the crystal structures of (1:1) complexes of dihomooxacalix[4]arenes with xylene isomers(3) and we tried to simulate the specific molecular recognition mechanism exhibited 
between the host and guest molecules by the computational method (electrostatic surface charge distribution, heat of formation and so on) and we found the important contribution of $\mathrm{CH}-\pi$ interactions which probably act to stabilize the guest molecule inside the cavity of host molecule.

(1)C. D. Gutsche et al., J. Amer. Chem. Soc., 103, 3782 (1981).

(2)J. Vicens et al., J. Inc. Phenom. 10, 159 (1991).

(3)K. Suzuki et al., Chem. Letters, 1991, 1699.

K. Suzuki, Doctor thesis, Osaka University (1991).

A. E. Armah et al., Acta Cryst., C48, 1474 (1992).

MS11.01.06 INCLUSION COMPOUNDS: RELATING STRUCTURE TO THERMAL STABILITY. L. R. Nassimbeni, Chemistry Department, University of Cape Town, Rondebosch 7700 , South Africa

Research on inclusion compounds has largely concentrated on the synthesis of novel host compounds, aimed at selective enclathration of targeted guest molecules. We have directed our program to the understanding of the intermolecular forces which are responsible for the process of molecular recognition between host and guest, in order to explain the physical properties of inclusion compounds.

We have synthesised a variety of host-guest complexes comprising bulky hosts containing the hydroxyl moiety, have elucidated their crystal structures and measured their thermodynamic stabilities with a variety of analytical techniques. The kinetics of enclathration of a gaseous guest by a solid host has been studied using a novel magnetic suspension balance, while the kinetics of thermal decomposition of labile clathrates has allowed us to elucidate the mechanism of the desolvation reactions. Competition experiments between close isomers of a guest have been carried out both in solution and with the host in suspension, and we have extended this principle to solid-solid systems.

The results of both the dynamic and equilibrium experiments are rationalised in terms of the molecular structures and the crystal packings of the host-guest compounds concerned.

MS11.01.07 MOLECULAR RECOGNITION OF ANIONS: HYDROGEN-BONDING PROPERTIES OF SULFATE, THIOCYANATE AND PICRATE ANIONS. Claudine Pascard, ICSN-CNRS 91198 Gif-sur-Yvette, France

Ions play an important role in biological systems, as substrates (ATP, peptidic side-chains..) or as structural elements at interfaces (membranes, micelles). Whereas the environment of cations is well studied, our goal is to analyse the structural characteristics of anion binding, very little 1 being written on this topic (aside of halogens).

The anions we started to study are geometrically very different: spherical, (sulfate dianion), linear (SCN) and flat (picrate). Sulfate plays a role in protein architecture, $\mathrm{SCN}$ has 2 acceptor centers and is very small, and picrate has a flat aromatic (hydrophobic) core. The method used was the search through Cambridge Crystallographic Data Bank, and we analysed the H-bonding possibilities of each anion observed in the retrieved crystal structures.

Discrete sulfate dianion principal characteristic is to form as many H-bonds as possible, short and direct, up to 12 per dianion. This quality gives sulfate a character of strong linker in forming molecular associations. The O-bonded sulfate (monoanion) is much less attractor and more directional. The $\mathrm{H}$-binding property of $\mathrm{SO}_{4}{ }^{2-}$ will depend on its complexing environment. SCN- has a very unusual behaviour. To our knowledge, this systematic analysis of its binding properties is the first one based on experimental results: isolated, this linear anion binds by both ends to H-donors: when coordinated to a metal by one end, it can binds to $\mathrm{H}$-donors by the other end. Metal cations fall into different classes whether they bind to $S$ or to N. Correlations between the charge (or size) of the cation and its position with respect to the anionic cylinder were found. Picrate forms strong hydrogen bonds which might involve the ortho nitro groups. The most evident result is the formation of intimate ion pairs. Other observed aggregation modes are infinite chains and macrorings.

The structural data assembled in these analyses can be useful to predict molecular associations, and in certain cases (sulfatel), agree quite well with similar analyses in biological systems. Moreover, our results provide the first picture of the coordination behaviour of SCN-.

(1)P. Chakrabarti (1993) J. Mol. Biol., 234. 463-482;

(2)R. S. Alexander, Z. F. Kanyo, L. E. Chirlian and D. W. Christianson (1990) J. A. C. S. 112, 933-937.

PS11.01.09 MODEL STUDY OFTHE RECOGNITION CENTER INVOLVING PYRIMIDINE BASES AND AMIDE GROUP OF AMUNO ACIDS. I.K. Galetich and V.S. Shelkovsky, Institute for Low Temperature Physics and Engineering of the Academy of Sciences of Ukraine

In the frames of the problem of molecular recognition this work is aimed at the investigation of hydrogen bond formation of the amide group with nucleic acid bases in the crystal structure of a specific repressor-operator complex of bacteriofage 434 . The interactions between the monomers of nucleic acids and proteins, which lead to hydrogen bonds formation, were studied on the systems (consisting of nitrogen bases 1,3-dimetUra and 1-metThy and acrylamide) which are good models of interactions, involving specific aminoacid side chains of Gln and Asn and free atomic groups of nucleic base Thy in single or double stranded DNA. Thermodynamic parameters of interactions were obtained using a method of temperature dependent field ionization mass spectrometry and theoretically by the atom-atom potential functions calculations. Under the mass spectrometric conditions the associates of molecules were formed in the gas phase; variation of the temperature allowed to obtain relative association constants and to calculate the enthalpies of associates formation (DH, kcal/mole) using Vant-Hoff plots. A good agreement between experimental and theoretical data for acrylamide complexes with 1,3-dimetUra $(9.7$ vs 10.1$)$ and 1 metThy ( 6.8 vs 6.6 ) was observed. Proposed schemes of the energetically favourable hydrogen bonding are as follows: a) for the complex of acrylamide with $1,3-\operatorname{dimetUra}: \mathrm{N} 1-\mathrm{H} . . \mathrm{O} 2 ; \mathrm{b}$ ) for the complex of acryl- amide with 1-metThy: O1 ...H-N2 or N1-H...O2. The data obtained enabled us to determine hydrogen bond for formation and mutual positions of molecules in the crystals and can be used for quantitative estimates of the probability of recognition.

PS11.01.10 MOLECULAR RECOGNITION OF THE PHY. TOHORMONE AUXIN AND RELATED COMPOUNDS. B. Kojic-Prodic, S. Antolic, S. Tomic and V. Magnus, Rudjer Boskovic Institute, P.O.B. 1016, 10001 Zagreb, Croatia

A molecular recognition approach was applied to the auxin family (indol-3-yl acetic acid, IAA), the most intensively studied plant hormone. The final goal of the research is to offer a mechanism of hormone binding to receptor(s) and to provide data for QSAR of indole and non-indole auxins. The study includes natural and synthetic alkylated and halogenated analogues of IAA. Both, active and inactive analogues have been included in the analyses. The problem has been tackled in three directions: analysis of the molecular geometry, study of electron redestribution by substitution effects, and correlation of the physico-chemical parameters with substitution effects and bioactivity. The analysis has been based on the results of X-ray structure analysis, computational chemistry, FT-IR, UV \& NMR spectroscopies, physico-chemical parameters such as lipophilicity and acid-base properties of the 